\title{
Comparing Spatially Distributed and Single Electrode Stimulation on Individuals with Spinal Cord Injury
}

\author{
Lucas De Macêdo Pinheiro ${ }^{1}$, Ana Carolina Cardoso De Sousa ${ }^{1}$ and Antônio Padilha Lanari Bó ${ }^{1,2}$
}

\begin{abstract}
It is still a challenge to delay the onset of fatigue on muscle contraction induced by Functional Electrical Stimulation (FES). We explored the use of two stimulation methods with the same total area, single electrode stimulation (SES), and spatially distributed electrical stimulation (SDSS) during isometric knee extension with spinal cord injured (SCI) volunteers. We applied stimulation on the left and right quadriceps of two SCI participants with both methods and recorded isometric force and evoked electromyography (eEMG). We calculated the force-time integral (FTI) and eEMG-time integral (eTI) for each stimulation series and used a linear regression as a measure of decay ratio. Moreover, we also estimated the contribution from each channel from eEMG.
\end{abstract}

\section{INTRODUCTION}

Individuals with neuromuscular disorders, such as spinal cord injury (SCI) and cerebral palsy, may experience a reduction of muscle mass and weakness. Functional electrical stimulation (FES) is a technique widely used to revert and prevent these consequences through induced muscle contractions achieved by the application of electric pulses [1].

The conventional single electrode stimulation (SES) consists of a pair of electrodes, often on the skin over the targeted muscles, from where current flows in and out at a specific frequency. On the other hand, we naturally achieve voluntary contraction of healthy muscles through selective, non-synchronous recruitment and activation of motor units [1]. Different from natural contractions, the static parameters, spatially fixed electrodes and synchronous activation in SES recruit the same fibers at a constant frequency, which can lead to increased fatigue and early force decay. Studies exploring ways to delay and reduce muscle fatigue [2]-[5] could have a significant impact in notably affected situations, e.g. intensive FES exercise [6] and co-activation application [7].

Spatially distributed sequential stimulation (SDSS) is a method that can generate muscle contractions resembling a physiological response by alternately activating different fibers [3]. Instead of stimulating at a specific frequency with

\footnotetext{
*This work was conducted as part of Empowering Mobility \& Autonomy Project (EMA Project) and supported by Coordenação de Aperfeiçoamento de Pessoal de Nível Superior (CAPES) and Conselho Nacional de Desenvolvimento Científico e Tecnológico (CNPq).

${ }^{1}$ L. M. Pinheiro, A. C. C. Sousa, and A. P. L. Bó are with Department of Electrical Engineering, University of Brasilia, Campus Darcy Ribeiro, Brazil. \{lucasdemacedo, anacsousa, antonio.plb\}elara.unb.br

${ }^{2}$ A. P. L. Bó is also with School of Information Technology and Electrical Engineering, The University of Queensland, Australia.
}

one large electrode, four smaller ones are placed side by side, as a $2 \times 2$ matrix, and the frequency is reduced to one fourth per electrode. This way, each pulse can generate a different electric field and activate less motor units at a lower rate, which allows for a reduced energy cost, delayed fatigue, and equal or greater power output [5]. Although muscles still fatigue, SDSS is more effective than SES in fatigue reduction [3]. Studies have presented similar strategies in distinct contexts, such as foot drop [8], and cycling [9], [10].

In [5], they compared SES and SDSS on the same leg, finding that SDSS yielded a higher power output in all tests. In experiments with knee flexors/extensors and plantar/dorsiflexors [3], SDSS demonstrated higher torque values for all $17 \mathrm{SCI}$ and 11 non-disabled participants, except for knee flexors. Furthermore, fatigue resistance was higher with SDSS in all muscle groups for all SCI individuals.

Previous works have focused on measuring the force signal [3], [4], which may accurately represent the muscle strength during an isometric exercise. However, when we apply SDSS, some muscle fibers may contribute more than others to the exercise, or even fatigue faster. We could consider this difference when designing FES applications and adjust the stimulation parameters in real-time for each channel to achieve greater efficiency.

We hypothesize that the electromyography (EMG) activity evoked by the electrical stimulation (eEMG) may provide each channel contribution. Therefore, the goal of the presented study is to investigate whether the eEMG may also show that the SDSS decreases muscle force decay over time when compared to SES during isometric contraction. Further, we also intend to illustrate that eEMG may estimate the contribution to each channel.

\section{METHODS}

\section{A. Participants}

We conducted the experiments with two SCI participants (P1 and P2), one man (41 years, $69 \mathrm{~kg}$, T9 injury level, and 6 years post-injury) and one woman (35 years, $54 \mathrm{~kg}$, T2 injury level, 5 years post-injury). P1 had well-trained muscles and regular use of electrical stimulation. Every participant provided written consent and agreed to participate. A local ethics committee ${ }^{1}$ approved the experimental tests.

\section{B. Data Acquisition}

Participants sat in an adjustable chair with one of their ankles fastened to a custom-made calibrated force sensor

${ }^{1}$ CAAE: 11717119.3 .0000 .0030$. 
keeping them from extending their leg and a support restrained their knee at $45^{\circ}$. The sensor consisted of a 3D printed structure with an electric circuit for signal conditioning, a microcontroller (Teensy 3.2, PJRC.COM LLC, USA), and a velcro strap hooked to a load cell (SEN-10245, Sparkfun Electronics, USA). We also recorded muscle activity with one EMG amplifier channel (RehaIngest, Hasomed GmbH, Germany) using $24 \mathrm{~mm}$ diameter $\mathrm{Ag} / \mathrm{AgCl}$ surface electrodes (Covidien, Dublin, Ireland) positioned on the rectus femoris as in Fig. 1 with the reference electrode on the participant's patella.

\section{Stimulation}

To perform the electrical stimulation, we used an 8-channel stimulator (RehaStim, Hasomed GmbH, Germany) and self-adhesive electrodes (Carcitrode, CARCI, Brazil) placed over the quadriceps. In both SES and SDSS methods, we applied stimulation with rectangular biphasic balanced pulses, current amplitude of $40 \mathrm{~mA}$, and pulse width set according to the setup phase, detailed in Section II-D.

In SES, we applied the stimulation on the $\mathrm{m}$. vastus lateralis, medialis and rectus femoris with two $9 \times 5 \mathrm{~cm}$ for both the active and reference electrodes and only one channel operating at $48 \mathrm{~Hz}$. Whereas, in SDSS, we used a group of four $4.5 \times 2.5 \mathrm{~cm}$ and a single $9 \times 5 \mathrm{~cm}$ electrode placed on the same muscle group. We used four stimulator channels, each at $12 \mathrm{~Hz}$ (48 divided by 4) as illustrated in Fig. 1. To share the reference between them, we made a 4 to 1 adaptor. The overall electrode area remained the same in both methods.

We used a computer with Robot Operating System (ROS) as a framework to integrate and operate the whole system ${ }^{2}$. We developed an application to run the experiment by communicating with the stimulator and the force sensor using the ROS client library for Python programming language.

\section{Protocol}

We divided the experiment into two main sessions for each participant, with a minimum of 24 hours in between so the participant could rest. Both legs were stimulated with SDSS in the first session and with SES in the second. As displayed

${ }^{2}$ For more information about the framework: http://www.ros.org in Fig. 2, each session was a sequence consisted of a setup phase, a rest period of $5 \mathrm{~min}$, and a stimulation phase of $6 \mathrm{~min}$ for each leg. The first leg assessed in each session was chosen at random.

Regarding the placement of SDSS electrodes, we chose a distal positioning for the four small electrodes since there was evidence of higher mean power output compared to proximal [2]. We determined the position by observing the muscle appearance and the force exerted. A satisfactory position was characterized by an evident contraction of the quadriceps. We used the same electrode placement in the second session.

In the setup phase, we manually increased the pulse width until the exerted force reached $20 \mathrm{~N}$. When we determined the pulse width and electrode position, the participant rested for $5 \mathrm{~min}$ and, after that, we started the stimulation. We recorded EMG and force throughout the procedure. At the end, we adjusted the chair and the sensor to repeat the sequence with the contralateral leg.

In the second session, we repeated the experiment with the same participant but with the SES method instead. So the procedure was the same besides placing the electrodes and making the proper adjustments to the stimulator frequency and channels. After five months we repeated the protocol described here for each participant, what we called second set. The first set refers to the first experiments.

\section{E. Data analysis}

For every stimulation phase of $6 \mathrm{~min}$, we normalized the force dividing it by the maximum value not corresponded to a spasm. A sudden contraction based on the muscle's visual aspect and an abrupt increase in force is what we considered as a spasm. We decided to normalize the data because it facilitates a direct comparison between force plots, as in Fig. 3.

There are several metrics to evaluate muscular force decay in time. For instance, in [2], [3], and [5] a fatigue index describes the percentage reduction in power or torque from the initial to the final phase. In our work, we calculated the area below the normalized force separately for each of the $5 \mathrm{~s}$ stimulation periods and fitted the area values to a linear model. We called the area as force-time integral (FTI),

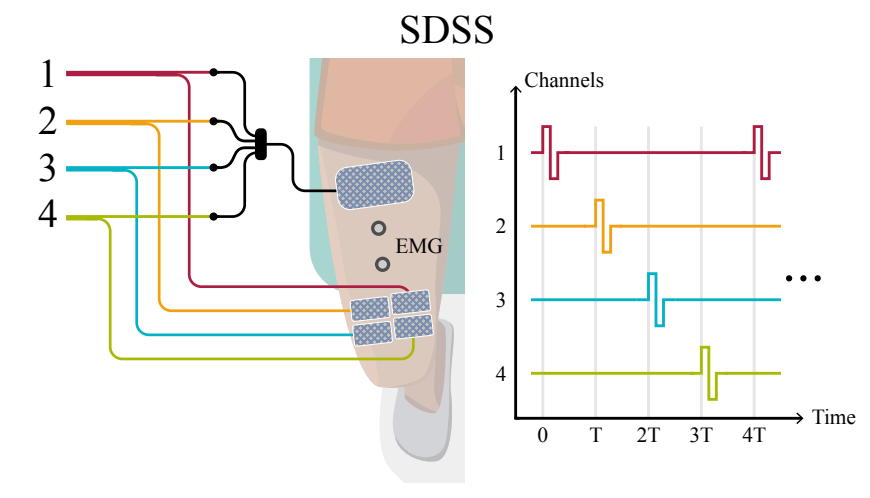

Fig. 1. SES and SDSS implementation and their respective setup.

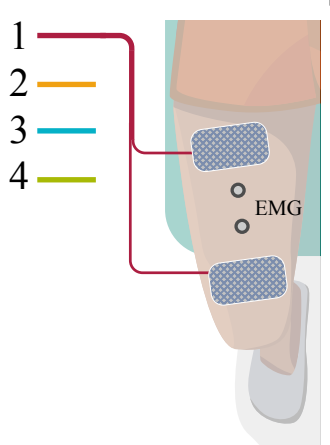

SES

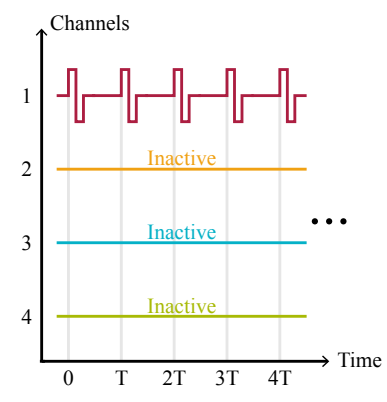

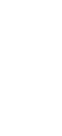




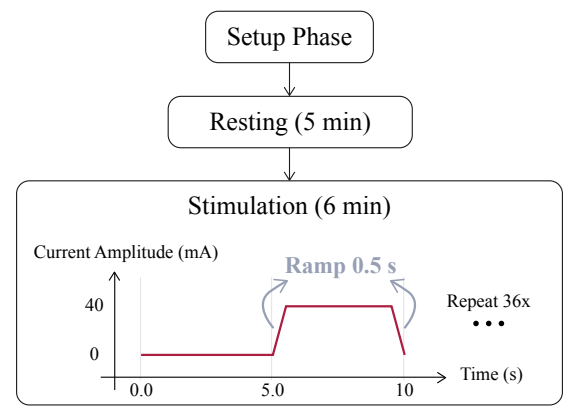

Fig. 2. In each session we performed this sequence of events twice, once for each leg. Two sessions, one of SDSS and one of SES, represent one set.

indicated by the right axis of Fig. 3 charts as an example. The slope of each regression was our measure to evaluate the force decay. During the isometric contraction, sometimes, the first sequences may provide much higher force response. Therefore, to also represent the response over time, we calculated the average of all FTI values $\left(\mathrm{FTI}_{\text {mean }}\right)$. A similar approach was used with torque in [11].

Moreover, with a single EMG channel we estimated the muscle activity (eEMG) evoked by each electrical stimulation channel using the artifact detection and filtering tools described in [12]. Similarly to the force response, we also calculated the eEMG-time integral (eTI), the linear regression and $\mathrm{eTI}_{\text {mean }}$ for each stimulation series.

\section{RESUlts}

Fig. 3 represents the force data acquired during one of the sessions, as well as, the FTI values and respective regression lines. In every experiment performed, FTI regressions had

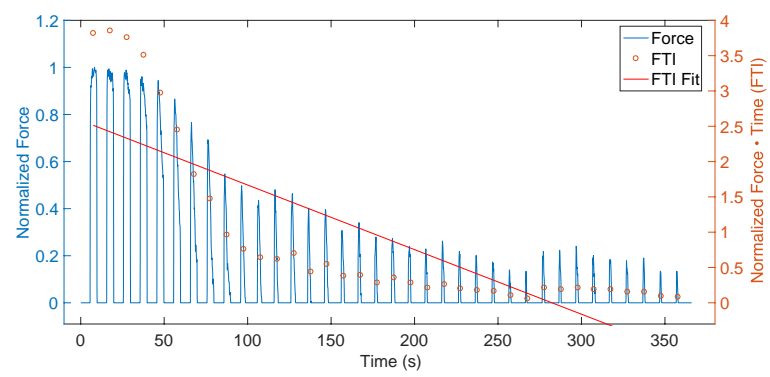

(a)

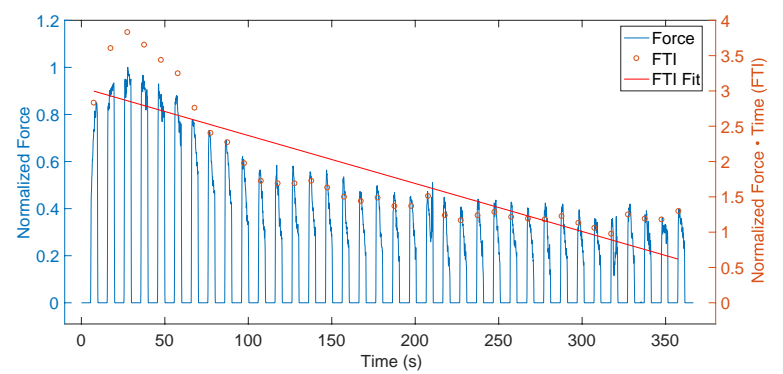

(b)

Fig. 3. First set of participant P1 left leg outcomes. The left axis refers to the normalized force and the right displays both the force-time integral (FTI) samples and their respective linear fit. SES in (a) and SDSS in (b). negative slopes, representing the force reduction throughout the stimulation phase. A less negative slope reflected a slower force decay. We used the linear model only as a decay metric, as the fatigue model is out of the scope of this work.

We may observe from Table I that most results from P1 led to slower fatigue (fitting slopes less negative) with SDSS, except for the right leg in the first set. P2 had an inverse result, the only exception being the right leg in the first set. In the second set, only the left leg of P2 had an inverse result. $\mathrm{FTI}_{\text {mean }}$ was lower for SES in all cases, except for the right leg of participant P2 in the first set. FTI mean was $84.34 \%$ higher with SDSS for P1 in the first set and $27.98 \%$ for P2 in the second. Pulse width with SDSS was lower than SES in all sessions.

During SDSS, we set a lower pulse width to achieve the same level of force as in SES, which means less stimulation intensity was required for a similar initial peak force. In addition, most of the $\mathrm{FTI}_{\text {mean }}$ values were higher, indicating a comprehensive greater area below the force profile. Hence, both the lower pulse width and higher FTI $_{\text {mean }}$ indicated greater overall stimulation efficiency.

\section{Discussion}

The accelerated muscle fatigue still is the primary challenge for FES control, and the possibility of selecting muscle fibers through eEMG information in FES control would cause a compelling impact on this topic. Like previous works [3]-[5], in our study, muscle activity data from SDSS suggested a slower decay on average when compared to SES. In our experiments, SDSS indicated more efficiency by having a mechanical output similar to SES at lower stimulation intensity. However, more research on the topic is still required as there are conflicting results regarding force decay.

During trials, we kept the EMG electrode positioning similar to both SES and SDSS, and this could explain the nominal values differences between force and EMG from Table I. Nevertheless, there are some similarities between the signals' response over time. Most of the results for both FTI

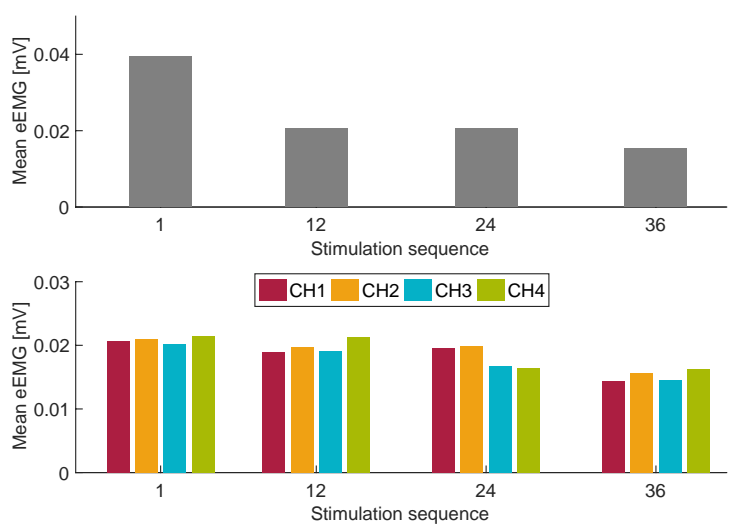

Fig. 4. The mean eEMG amplitude for SES (top) and for each stimulation channel of SDSS (bottom) during the second set of participant P1 left leg session. 
TABLE I

EEMG AND FORCE-TIME INTEGRAL OUTCOMES

\begin{tabular}{|c|c|c|c|c|c|c|c|c|c|c|c|c|c|c|c|c|c|}
\hline & & \multicolumn{4}{|c|}{ FTI Fitting Slope * } & \multicolumn{4}{|c|}{ FTI mean } & \multicolumn{4}{|c|}{ eTI Fitting Slope * } & \multicolumn{4}{|c|}{ eTI mean } \\
\hline & & \multicolumn{2}{|c|}{ 1st Set } & \multicolumn{2}{|c|}{ 2nd Set } & \multicolumn{2}{|c|}{ 1st Set } & \multicolumn{2}{|c|}{ 2nd Set } & \multicolumn{2}{|c|}{ 1st Set } & \multicolumn{2}{|c|}{ 2nd Set } & \multicolumn{2}{|c|}{ 1st Set } & \multicolumn{2}{|c|}{ 2nd Set } \\
\hline & & SES & SDSS & SES & SDSS & SES & SDSS & SES & SDSS & SES & SDSS & SES & SDSS & SES & SDSS & SES & SDSS \\
\hline \multirow{3}{*}{ P1 } & $\mathrm{L}$ & -9.147 & -6.796 & -6.094 & -3.153 & 0.913 & 1.808 & 1.973 & 2.956 & -3.874 & -1.115 & -4.061 & -0.614 & 1.075 & 1.142 & 1.282 & 1.288 \\
\hline & $\mathrm{R}$ & -7.223 & -7.539 & -7.511 & -3.975 & 1.418 & 2.489 & 1.457 & 3.202 & -2.264 & -2.495 & -1.624 & -1.007 & 0.912 & 0.549 & 0.656 & 0.881 \\
\hline & Mean & -8.185 & -7.168 & -6.803 & -3.564 & 1.166 & 2.149 & 1.715 & 3.079 & -3.069 & -1.805 & -2.843 & -0.811 & 0.994 & 0.846 & 0.969 & 1.085 \\
\hline \multirow{3}{*}{$\mathrm{P} 2$} & $\mathrm{~L}$ & -5.058 & -6.084 & -6.047 & -6.464 & 2.027 & 2.602 & 1.667 & 2.203 & - & -0.185 & -0.254 & 0.407 & - & 1.753 & 1.519 & 0.717 \\
\hline & $\mathrm{R}$ & -5.613 & -5.548 & -8.576 & -8.549 & 1.776 & 1.720 & 1.621 & 2.005 & -1.401 & -0.481 & 0.703 & 0.477 & 2.244 & 1.496 & 1.358 & 1.766 \\
\hline & Mean & -5.336 & -5.816 & -7.312 & -7.507 & 1.902 & 2.161 & 1.644 & 2.104 & -1.401 & -0.333 & 0.225 & 0.442 & 2.244 & 1.6245 & 1.439 & 1.242 \\
\hline
\end{tabular}

${ }^{*}$ The Fitting Slope is on a $10^{-3}$ scale.

The " - " represents missing data. Abbreviations: P1, participant 1; P2, participant 2; L, left leg; R, right leg; FTI, Force-Time Integral; eTI, eEMG-Time Integral; SES, single electrode stimulation; SDSS, spatially distributed sequential stimulation.

and eTI fitting slopes show that, as expected, SDSS presented slower fatigue when compared to SES of the same subject, leg, and trial (especially for P1).

Moreover, from Fig. 4, we may observe that most channels presented similar eEMG amplitude at each sequence. Over time, channels 1 and 3 seem to fatigue faster when compared to channels 2 and 4 based on observations in participant P1's left leg. We believe that it would be possible to adjust these signals in real-time with a controller, possibly improving performance in terms of fatigue resistance.

\section{CONCLUSION}

To our knowledge, we have implemented the first evoked muscle activity (eEMG) estimation for each channel of the SDSS method, which could lead to more selectively controllers. In our protocol, the force data indicated greater overall efficiency and the eEMG suggested slower mean muscle activity decay for both subjects when comparing SDSS with SES. Additional research with more participants to explore different fitting models and measures is indispensable to consolidate these findings. Future work could compare muscle activity along with doublet and triplet electrical pulses used in combination with SES and SDSS. As in [13], we could explore the effects of different stimulation waveforms as well. Additionally, other applications, such as FES cycling, could also be useful for investigation.

\section{REFERENCES}

[1] D. B. Popovic and T. Sinkjær, Control of movement for the physically disabled: control for rehabilitation technology. Springer, 2000.

[2] M. Laubacher, E. A. Aksöz, S. Binder-Macleod, and K. J. Hunt, "Comparison of proximally versus distally placed spatially distributed sequential stimulation electrodes in a dynamic knee extension task," European journal of translational myology, vol. 26, no. 2, 2016.

[3] D. G. Sayenko, R. Nguyen, T. Hirabayashi, M. R. Popovic, and K. Masani, "Method to reduce muscle fatigue during transcutaneous neuromuscular electrical stimulation in major knee and ankle muscle groups," Neurorehabilitation and neural repair, vol. 29, no. 8, pp. 722-733, 2015.
[4] N. M. Malešević, L. Z. Popović, L. Schwirtlich, and D. B. Popović, "Distributed low-frequency functional electrical stimulation delays muscle fatigue compared to conventional stimulation," Muscle \& nerve, vol. 42, no. 4, pp. 556-562, 2010.

[5] M. Laubacher, E. A. Aksoez, A. K. Brust, M. Baumberger, R. Riener, S. Binder-Macleod, and K. J. Hunt, "Stimulation of paralysed quadriceps muscles with sequentially and spatially distributed electrodes during dynamic knee extension," Journal of neuroengineering and rehabilitation, vol. 16, no. 1, pp. 1-12, 2019.

[6] A. P. Bo, L. O. da Fonseca, J. A. Guimaraes, E. Fachin-Martins, M. E. Paredes, G. A. Brindeiro, A. C. C. de Sousa, M. C. Dorado, and F. M. Ramos, "Cycling with spinal cord injury: A novel system for cycling using electrical stimulation for individuals with paraplegia, and preparation for cybathlon 2016," IEEE Robotics \& Automation Magazine, vol. 24, no. 4, pp. 58-65, 2017.

[7] A. P. L. Bo, L. O. da Fonseca, and A. C. C. de Sousa, "Fes-induced coactivation of antagonist muscles for upper limb control and disturbance rejection," Medical engineering \& physics, vol. 38, no. 11, pp. 1176$1184,2016$.

[8] J. Malešević, S. D. Dujović, A. M. Savić, L. Konstantinović, A. Vidaković, G. Bijelić, N. Malešević, and T. Keller, "A decision support system for electrode shaping in multi-pad fes foot drop correction," Journal of neuroengineering and rehabilitation, vol. 14, no. 1 , p. 66, 2017.

[9] M. Laubacher, E. A. Aksöz, I. Bersch, and K. J. Hunt, "The road to cybathlon 2016-functional electrical stimulation cycling team irpt/spz," European journal of translational myology, vol. 27, no. 4, 2017.

[10] R. J. Downey, T.-H. Cheng, M. J. Bellman, and W. E. Dixon, "Switched tracking control of the lower limb during asynchronous neuromuscular electrical stimulation: Theory and experiments," IEEE transactions on cybernetics, vol. 47, no. 5, pp. 1251-1262, 2016.

[11] V. Rozand, T. Cattagni, J. Theurel, A. Martin, and R. Lepers, "Neuromuscular fatigue following isometric contractions with similar torque time integral," International journal of sports medicine, vol. 36 , no. 01, pp. 35-40, 2015.

[12] A. C. C. de Sousa, M. Valtin, A. P. Bó, and T. Schauer, "Automatic detection of stimulation artifacts to isolate volitional from evoked emg activity," IFAC-PapersOnLine, vol. 51, no. 27, pp. 282-287, 2018.

[13] F. V. Medeiros, M. Bottaro, A. Vieira, T. P. Lucas, K. A. Modesto, A. P. L. Bo, G. Cipriano Jr, N. Babault, and J. L. Q. Durigan, "Kilohertz and low-frequency electrical stimulation with the same pulse duration have similar efficiency for inducing isometric knee extension torque and discomfort," American journal of physical medicine \& rehabilitation, vol. 96, no. 6, pp. 388-394, 2017. 